\title{
PRESSÃO DE PRECONSOLIDAÇÃO E INTERVALO HÍDRICO ÓTIMO COMO INDICADORES DE ALTERAÇÕES ESTRUTURAIS DE UM LATOSSOLO E DE UM CAMBISSOLO SOB CANA-DE-AÇÚCAR ${ }^{(1)}$
}

\author{
Eduardo da Costa Severiano ${ }^{(2)}$, Geraldo César de Oliveira ${ }^{(3)}$, Moacir \\ de Souza Dias Júnior ${ }^{(4)}$, Luiz Fernando Coutinho de Oliveira ${ }^{(5)} \&$ \\ Matheus Bornelli de Castro ${ }^{(6)}$
}

\begin{abstract}
RESUMO
A identificação dos impactos da colheita mecanizada da cultura da cana-deaçúcar sobre a estrutura do solo em diferentes classes pode-se tornar uma importante estratégia de manejo para a minimização dos efeitos da compactação. O objetivo deste trabalho foi avaliar as alterações estruturais, a pressão de preconsolidação e o intervalo hídrico ótimo (IHO) em um Latossolo VermelhoAmarelo (LVA) e um Cambissolo Háplico (CX), em conseqüência das operações de colheita da cana-de-açúcar em diferentes épocas de safra. Coletaram-se amostras indeformadas de solo na profundidade de 0 a $0,05 \mathrm{~m}$ para determinar a pressão de preconsolidação, a densidade, a porosidade do solo, a agregação e o IHO, bem como amostras deformadas para a caracterização química e física do solo. $\mathrm{O}$ CX apresentou maior resistência à compactação que o LVA. O conteúdo de água no solo durante a colheita da cana-de-açúcar variou na ordem: agosto de 2006 < novembro de 2005 < março de 2006 e CX > LVA em cada época. A densidade do solo na pressão de preconsolidação foi sensível à ocorrência de alterações estruturais no solo. A macroporosidade limitante apresentou restrições ao
\end{abstract}

\footnotetext{
(1) Parte da Dissertação de Mestrado do primeiro autor, apresentada à Universidade Federal de Lavras - UFLA. Projeto financiado pelo Conselho Nacional de Desenvolvimento Científico e Tecnológico (CNPq) e Empresa Jalles Machado S.A. Recebido para publicação em setembro de 2007 e aprovado em maio de 2008.

(2) Doutorando em Ciência do Solo, Departamento de Ciência do Solo da Universidade Federal de Lavras - DCS/UFLA. Caixa Postal 37, CEP 37200-000 Lavras (MG). Bolsista do CNPq. E-mail: severianoec@yahoo.com.br

(3) Professor Adjunto, DCS/UFLA, E-mail: geraldooliveira@ufla.br

(4) Professor Associado, DCS/UFLA. Bolsista do CNPq. E-mail: msouzadj@ufla.br

(5) Professor Associado, Departamento de Engenharia, UFLA. Bolsista do CNPq. E-mail: lfco@pq.cnpq.br

${ }^{(6)}$ Graduando em Agronomia, UFLA. Bolsista de PIBIC/CNPq. E-mail: matheusbcastro@ig.com.br
} 
crescimento das plantas em níveis de compactação inferiores à densidade do solo crítica do IHO. A colheita mecanizada da cana-de-açúcar realizada em solo com 0,16 e $0,21 \mathrm{~kg} \mathrm{~kg}^{-1}$ de água, para LVA e CX, respectivamente, não promoveu compactação excessiva.

Termos de indexação: Saccharum sp., mecanização da colheita, estrutura do solo.

\title{
SUMMARY: PRECONSOLIDATION PRESSURE AND LEAST LIMITING WATER RANGE AS INDICATORS OF STRUCTURAL CHANGES OF A LATOSOL AND A CAMBISOL UNDER SUGARCANE
}

\begin{abstract}
The identification of impacts of mechanized sugarcane harvesting on the structure in different soil classes can be an important strategy to minimize compaction effects. The objective of this study was to evaluate the structural changes, preconsolidation pressure and least limiting water range (LLWR) in a Red-Yellow Latosol (LVA) and a Haplic Cambisol (CX), due to harvest operations at different periods during the sugar cane harvesting season. Undisturbed soil samples were collected in the layer 0 to $0.05 \mathrm{~m}$ to determine preconsolidation pressure, bulk density, porous space, aggregation and the LLWR. Disturbed soil samples were taken for chemical and physical characterization of the soil. The compaction resistance of $C X$ was higher than that of LVA. The soil water content during the harvest of sugarcane varied in the following order: August $2006<$ November $2005<$ March 2006, and CX > LVA in each period. The preconsolidation pressure showed that bulk density was sensitive to occurrence of structural changes in the soil. Limiting macroporosity restricted plant growth at compaction levels below the critical bulk density of LLWR. Mechanized sugarcane harvesting at soil water contents of up to 0.16 and $0.21 \mathrm{~kg} \mathrm{~kg}^{-1}$, for LVA and CX, respectively, did not cause excessive compaction.
\end{abstract}

Index terms: Saccharum sp, harvest mechanization, soil structure.

\section{INTRODUÇÃO}

A técnica de produção de cana-de-açúcar tem demonstrado evolução no que se refere à utilização de máquinas, implementos, técnicas de manejo e transporte, com a opção por veículos de maior capacidade unitária de carga (Iaia et al., 2006), o que provoca, entretanto, impactos adversos no solo, destacando-se o processo de compactação. Em áreas canavieiras, a compactação tem ocorrido em virtude das operações de manejo que envolve o tráfego de máquinas em conteúdos inadequados de água no solo. Dessa forma, a mecanização da colheita pode influenciar a produção e a longevidade da cultura, os atributos físicos, químicos e biológicos do solo, resultando em danos ambientais (Souza et al., 2005).

A manutenção de condições físicas adequadas ao crescimento das plantas está relacionada com a preservação da estrutura do solo e pode ser alcançada por meio do conhecimento do comportamento compressivo do solo e monitoramento das operações mecanizadas. As alterações nas relações massa/ volume e na resistência mecânica do solo interagem entre si, alterando o ambiente radicular (Silva et al., 2001). Nesse contexto, o intervalo hídrico ótimo (IHO) tem sido sugerido como indicador de qualidade do solo que influenciam diretamente a produção das culturas por englobar em torno de uma única variável a resistência à penetração, a disponibilidade de água e a aeração, os quais alteram diretamente a produtividade agrícola (Silva et al., 1994).

A amplitude do IHO indica o risco de exposição das culturas ao estresse físico do solo e a magnitude na qual a condição estrutural restringe o crescimento e desenvolvimento das plantas (Silva \& Kay, 1997). Solos com estrutura preservada apresentam restrições apenas em termos de déficit hídrico. Entretanto, quando a compactação atinge níveis excessivos, a aeração se torna deficiente sob condições de elevados conteúdos de água, e a resistência à penetração pode restringir o crescimento das plantas com o secamento do solo (Lapen et al., 2004). Nesse cenário, o IHO significa grande avanço nos estudos de biofísica do solo, sendo o indicador que melhor se correlaciona com o crescimento das plantas (Tormena et al., 2007).

As diferenças no arranjamento das partículas do solo, derivadas da pedogênese (Centurion et al., 2007), resultam em comportamento diferenciado quando 0 solo é submetido à compressão decorrente do tráfego de máquinas. Assim, para um correto planejamento das atividades mecanizadas, é necessário o conhecimento do comportamento compressivo em 
diferentes classes de solo (Dias Júnior et al., 2007), baseados no manejo e na variação do conteúdo de água no solo (Oliveira et al., 2003). A minimização dos impactos na estrutura dependerá da adequação da trafegabilidade das máquinas sobre o solo em diferentes condições climáticas ao longo da safra canavieira.

Este trabalho objetivou avaliar a pressão de preconsolidação, o intervalo hídrico ótimo e as alterações estruturais de um Latossolo VermelhoAmarelo e um Cambissolo Háplico no município de Goianésia, GO, submetidos às operações de colheita da cana-de-açúcar em diferentes épocas da safra.

\section{MATERIAL E MÉTODOS}

Para a condução deste estudo, selecionaram-se duas áreas experimentais pertencentes à empresa Jalles Machado S.A., localizada no município de Goianésia, $\mathrm{GO}$, a $15^{\circ} 10^{\prime} \mathrm{S}$ de latitude, $49^{\circ} 15^{\prime} \mathrm{W}$ de longitude e $640 \mathrm{~m}$ de altitude. O clima local é do tipo Aw, segundo Köppen, quente e úmido, com longa estação seca e precipitações pluviais anuais médias de $1.500 \mathrm{~mm}$.

Os solos das áreas experimentais foram classificados, segundo Embrapa (2006), como Latossolo Vermelho-Amarelo argiloso (LVA), com $0,03 \mathrm{~m} \mathrm{~m}^{-1} \mathrm{de}$ declividade e pertencente à classe III do sistema de capacidade de uso, segundo classificação de Lepsch (1991), tendo como maior limitação a baixa fertilidade natural e um Cambissolo Háplico franco-argiloarenoso (CX), com $0,12 \mathrm{~m} \mathrm{~m}^{-1}$ de declividade, pertencente à classe IV do sistema de capacidade de uso, sendo a declividade do terreno o fator mais limitante para o manejo conservacionista.
Em março de 2004, procedeu-se à renovação do canavial, submetendo as áreas ao preparo do solo com uma aração e duas gradagens, sulcados por ocasião do plantio da cana-de-açúcar. Em setembro de 2005, foram demarcadas, em cada área, 12 parcelas com dimensões de $10 \mathrm{~m}$ de comprimento e $14 \mathrm{~m}$ de largura, com 10 linhas de cana-de-açúcar, com espaço de $1,40 \mathrm{~m}$.

Antes da realização da colheita da cana-de-açúcar, foram feitas amostragens em três pontos de uma linha diagonal dentro de cada parcela, distantes $6,6 \mathrm{~m}$ uma das outras, e pontos extremos a $2 \mathrm{~m}$ da borda limítrofe da parcela. Coletaram-se, na posição das entrelinhas da cana-de-açúcar, seis amostras indeformadas por parcela, na profundidade de 0 a $0,05 \mathrm{~m}$, em cilindros com dimensões de $6,40 \mathrm{~cm}$ de diâmetro e $2,50 \mathrm{~cm}$ de altura, totalizando 144 amostras (6 amostras x 12 parcelas $\mathrm{x} 2$ solos), as quais foram parafinadas no campo para a manutenção do conteúdo de água. Foram coletadas também quatro amostras deformadas na profundidade do horizonte diagnóstico de cada solo, para análise para classificação do solo. As amostras deformadas resultantes das sobras dos anéis foram submetidas à caracterização química e física dos solos (Quadro 1) e ao aparelho extrator de Richards à tensão de $1.500 \mathrm{kPa}$, para determinação do ponto de murcha permanente, segundo Embrapa (1997).

As amostras indeformadas foram inicialmente saturadas e submetidas à tensão de $6 \mathrm{kPa}$ para a determinação da microporosidade e capacidade de campo (Embrapa, 1997; Mello et al., 2002). Posteriormente, essas amostras foram ajustadas a conteúdos de água no solo, variando de 0,08 a $0,36 \mathrm{~kg} \mathrm{~kg}^{-1}$, e submetidas ao teste de penetrometria, utilizando um penetrômetro de bancada MARCONI,

Quadro 1. Caracterização química e física do Latossolo Vermelho-Amarelo (LVA) e Cambissolo Háplico (CX) cultivados com cana-de-açúcar, no município de Goianésia, GO

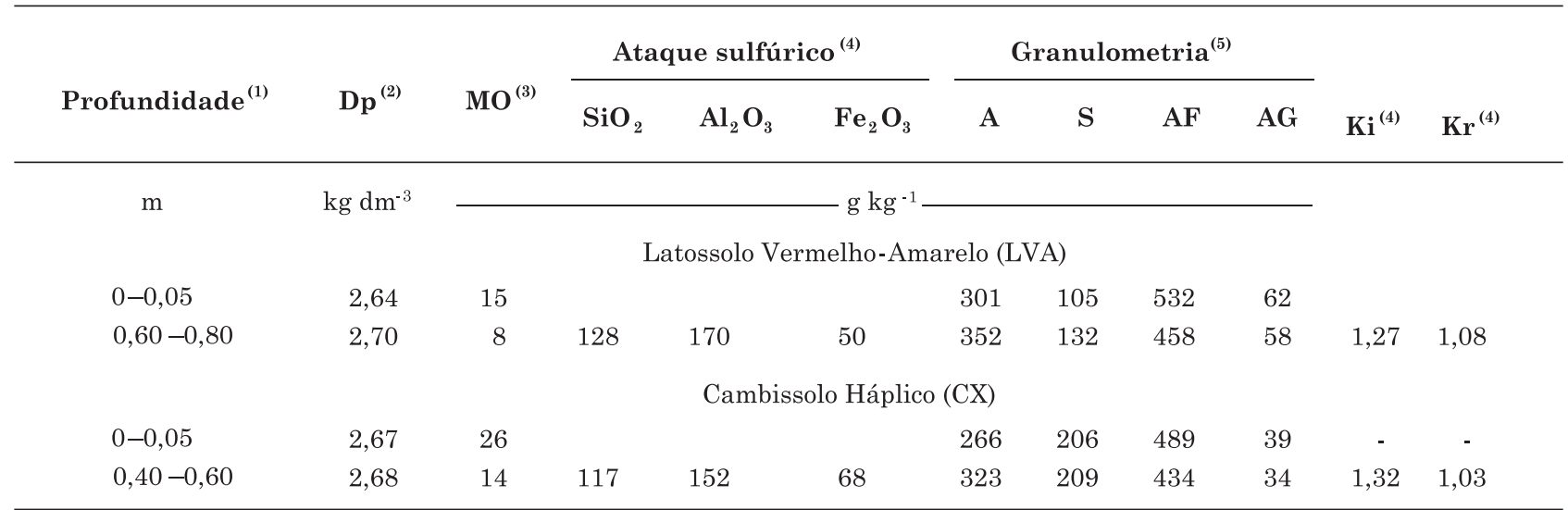

(1) Profundidades correspondentes à camada superficial e ao horizonte B (diagnóstico) dos solos. (2): Dp: densidade de partículas pelo método do picnômetro (média de 12 repetições). ${ }^{(3):}$ MO: matéria orgânica (média de 12 repetições) ${ }^{(4)}$ Média de quatro repetições, determinada apenas no horizonte diagnóstico dos solos. ${ }^{(5)}$ Determinada pelo método da pipeta (média de 12 repetições); A: argila; S: silte; AF: areia fina; AG: areia grossa; Ki: relação molecular $\mathrm{SiO}_{2}: \mathrm{Al}_{2} \mathrm{O}_{3} ; \mathrm{Kr}$ : relação molecular $\mathrm{SiO}_{2}:\left(\mathrm{Al}_{2} \mathrm{O}_{3}+\right.$ $\left.\mathrm{Fe}_{2} \mathrm{O}_{3}\right)$. 
modelo MA 933, dotado de variador eletrônico de velocidade e sistema de registro de dados, segundo Tormena et al. (1998), e ao ensaio de compressão uniaxial, usando um consolidômetro de marca Boart Longyear, cuja aplicação das pressões é feita por meio da utilização de ar comprimido, segundo método descrito por Dias Júnior (1994).

Em seguida, as amostras foram secas em estufa a $105^{\circ} \mathrm{C}$, por $48 \mathrm{~h}$, para determinação da densidade do solo (Ds). O volume total de poros (VTP) foi determinado pela expressão: $V T P=(1-D s / D p)$, sendo Dp a densidade de partículas. A macroporosidade do solo foi obtida pela diferença entre VTP e microporosidade, segundo Embrapa (1997). A pressão de preconsolidação $\left(\sigma_{p}\right)$ e a densidade do solo na pressão de preconsolidação (Ds $\left.\sigma_{\mathrm{p}}\right)$ foram obtidas da curva de compressão do solo segundo Dias Júnior \& Pierce (1995), utilizando uma planilha eletrônica de fluxo livre para determinação dos parâmetros da curva de compressão do solo. A relação das densidades $(\Delta \mathrm{Ds})$ foi obtida pela relação $D s / D s \sigma_{p}$.

Os valores de resistência à penetração $(\mathrm{RP})$, obtidos em $\mathrm{kgf} \mathrm{cm}^{-2}$, foram multiplicados pelo fator 0,098 para transformação em MPa e ajustados a modelos nãolineares, em função do conteúdo volumétrico de água ( $\theta$ ) e da Ds, segundo Busscher (1990). Empregandose as equações 1 e 2 , foram determinados os conteúdos de água no solo, cuja resistência à penetração assume valores limitantes ao crescimento e desenvolvimento das plantas $\left(\theta_{\mathrm{RP}}\right)$ :

$$
\begin{aligned}
& \text { LVA: } R P=0,005 \theta^{-2,22} D S^{6,01} ; \mathrm{R}^{2}=0,73^{* *} \\
& C X: R P=0,052 \theta^{-0,86} D S^{7,01} ; \mathrm{R}^{2}=0,70^{* *}
\end{aligned}
$$

Consideraram-se como limites superiores do IHO (Silva et al., 1994) o conteúdo de água no solo na capacidade de campo $\left(\theta_{\mathrm{CC}}\right) \mathrm{e}$, ou, a porosidade de aeração adequada ao desenvolvimento da cultura $\left(\theta_{\mathrm{PA}}\right)$, obtida pela fórmula: $\theta_{P A}=(V T P-0,1)($ Silva et al., 2001). Os limites inferiores foram considerados o conteúdo de água no ponto de murcha permanente $\left(\theta_{\mathrm{PMP}}\right)$ e, ou, o conteúdo de água correspondente à resistência à penetração de $2,5 \mathrm{MPa}\left(\theta_{\mathrm{RP}}\right)$ (Camargo \& Alleoni, 1997).

Para avaliação dos efeitos das operações de colheita da cana-de-açúcar sobre a estrutura do solo, foi realizada a colheita mecanizada, utilizando-se tráfego de duas passadas em cada entrelinha da cultura do seguinte maquinário: colhedora de cana Cameco, modelo CWH $2500 \mathrm{~B}$, com tara de 16,5 Mg; trator John Deere, modelo 6605, com tara de 4,5 Mg; e transbordo Cameco, modelo SMR 8500, com tara de $6,4 \mathrm{Mg}$, carregados com $8 \mathrm{Mg}$ de cana picada.

Procedeu-se à colheita em três parcelas de cada solo em cada época, nos meses de novembro de 2005, março e agosto de 2006, possibilitando que os solos se encontrassem em diferentes conteúdos de água por ocasião dessa operação. A colheita manual de cana sem tráfego de máquinas foi realizada em março de
2006, por ser esta a época de maior intensidade pluvial na região, para comparação com as prováveis alterações estruturais decorrentes da colheita mecanizada.

Após a colheita da cana-de-açúcar, coletaram-se amostras indeformadas e, no laboratório, procedeu-se à determinação do conteúdo de água no solo por ocasião da colheita (Embrapa, 1997), macro e microporosidade, teste de penetrometria e limites do IHO.

Coletaram-se, ainda, 144 amostras indeformadas (3 amostras x 12 parcelas x 2 solos x 2 épocas) para a obtenção de materiais de solo com diâmetro entre 2,0 e 8,0 mm, conforme recomendações de Guedes (1997). A estabilidade de agregados foi determinada por peneiramento em água, sendo utilizado o diâmetro médio geométrico (DMG) como índice de agregação (Kemper \& Rosenau, 1986). O teor de matéria orgânica (MO) (Raij \& Quaggio, 1983) foi determinado no material de solo inferior a 2,0 $\mathrm{mm}$ obtido no preparo das amostras dos agregados.

Para a obtenção dos modelos de capacidade de suporte de carga do solo, a pressão de preconsolidação $\left(\sigma_{\mathrm{p}}\right)$ foi ajustada em função do conteúdo gravimétrico de água (U), segundo Dias Júnior (1994), usando o software Sigma Plot 9.0. A comparação das regressões foi realizada seguindo os procedimentos descritos por Snedecor \& Cochran (1989).

Os IHOs foram obtidos ajustando-se os limites do conteúdo volumétrico de água em função da Ds (Silva et al., 1994). A densidade do solo crítica ao crescimento e desenvolvimento das plantas (Dsc) foi considerada como o valor de $\mathrm{Ds}$ em que o $\mathrm{IHO}=\mathrm{O}$. A água disponível (AD) foi considerada a diferença entre os conteúdos volumétricos de água na capacidade de campo $\left(\theta_{\mathrm{CC}}\right)$ e no ponto de murcha permanente $\left(\theta_{\mathrm{PMP}}\right)$, quando estes foram os limites superiores e inferiores do IHO.

Os resultados dos atributos físicos do solo foram submetidos à análise de variância, segundo delineamento em blocos casualizados e parcelas subdivididas no tempo, sendo estas as avaliações realizadas antes e após as operações de colheita. As comparações das médias foram realizadas pelo teste de Scott-Knott a $5 \%$.

\section{RESULTADOS E DISCUSSÃO}

O valor de Ki inferior a 2,2 no CX o caracteriza como sendo muito intemperizado (Embrapa, 2006; Quadro 1). Resende et al. (2007) justificam esses valores pela provável formação a partir do horizonte $\mathrm{C}$ de antigos Latossolos, cujos horizontes A e B foram erodidos em passado muito distante. Contudo, a presença do horizonte B incipiente, considerado como principal critério de classificação do referido sistema, caracteriza-o como Cambissolo. 
Os solos apresentaram comportamentos diferenciados em termos de capacidade de suporte de carga do solo (não homogêneo para as regressões, $\mathrm{F}=50,17 ; \mathrm{p}<0,001$ para o coeficiente linear $\mathrm{e}$ $\mathrm{F}=134,80 ; \mathrm{p}<0,001$ para o coeficiente angular). A maior capacidade de suporte de carga foi verificada no CX (Figura 1), o que está relacionado ao maior ajuste entre partículas provenientes de sua estrutura em blocos subangulares de classe pequena a média e grau moderado a forte (Santos et al., 2005), e pelo maior teor de MO (Quadro 1). Por outro lado, a estrutura do LVA, do tipo blocos subangulares, classe muito pequena, grau fraco de agregação e que se desfazem em grânulos, confere-lhe maior possibilidade de ajustes das partículas do solo quando submetido ao tráfego de máquinas, apesar da semelhança mineralógica (caulinítica, para valores de $\mathrm{Kr}$ superior a 0,75, segundo Embrapa, 2006) e textural (Quadro 1) entre os solos, citadas por Lebert \& Horn (1991) como fatores internos determinantes no processo de compactação.

A capacidade de suporte de carga dos solos estudados pode ser considerada baixa, sob altos conteúdos de água (Figura 1). Para o LVA, a redução da pressão de preconsolidação com o incremento do conteúdo de água ocorreu de maneira abrúptica, verificada pelo elevado coeficiente angular da regressão $(7,36)$. Esse comportamento pode ser atribuído à eliminação da história de tensão do solo (Kondo \& Dias Júnior, 1999) durante o seu preparo por ocasião da renovação do canavial, exigindo, portanto, maior cautela no momento adequado para a realização das operações mecanizadas de colheita, a fim de se evitar a degradação estrutural.

O incremento na densidade do solo inicial (Dsi) eleva a pressão de preconsolidação (Dias Júnior, 1994). Entretanto, apesar da maior Dsi no LVA (Quadro 2), o solo apresentou menor capacidade de suporte de carga (Figura 1). Esse comportamento é explicado pelas diferenças na estrutura pedológica dos dois solos, onde o tipo, a classe e o grau da estrutura, este último quantificado por meio da pressão de preconsolidação, sugerem que o estado de agregação do solo exerce maior influência que a Dsi no seu comportamento compressivo, quando são comparados solos distintos.

A realização da colheita da cana-de-açúcar em diferentes épocas promoveu o tráfego das máquinas em diferentes conteúdos de água no solo (Quadro 3). Em função do regime pluvial da região, o incremento no conteúdo de água dos solos deu-se na seguinte ordem: agosto de 2006 < novembro de $2005<$ março de 2006. Por outro lado, o CX apresentou maiores conteúdos de água em todas as épocas avaliadas, o que poderia ser um fator determinante nas alterações estruturais, dada a relevância da umidade no processo de compactação do solo (Dias Júnior, 1994).

As alterações estruturais nos solos provocadas pelas operações de colheita mecanizada da cana-de-açúcar ocorreram em função da capacidade de suporte de carga nas diferentes épocas (Figura 1 e Quadro 3). A colheita manual de cana sem tráfego de máquinas

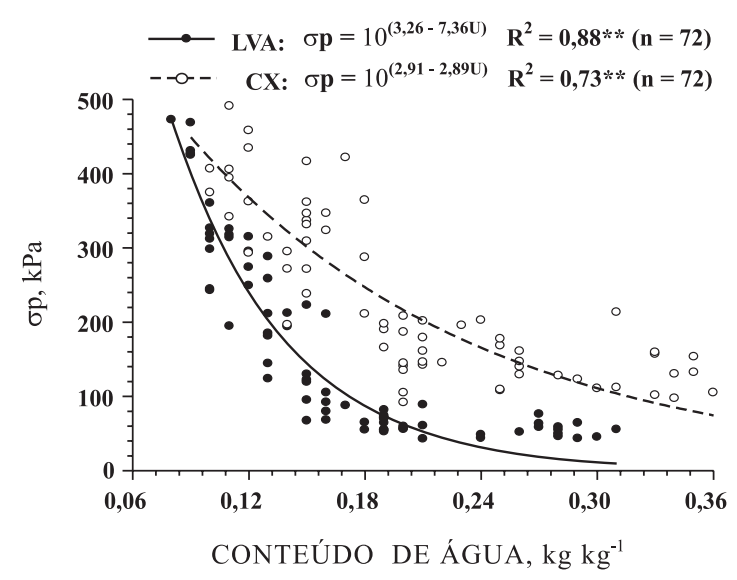

Figura 1. Modelos de capacidade de suporte de carga [variação da pressão de preconsolidação $\left(\sigma_{p}\right)$ em função do conteúdo de água] do Latossolo Vermelho-Amarelo (LVA) e do Cambissolo Háplico (CX), na profundidade de 0-0,05 m.

Quadro 2. Volume total de poros (VTP), densidade (Ds), densidade na pressão de preconsolidação (Ds $\left.\sigma_{\mathrm{p}}\right)$ e a relação dessas densidades $(\Delta \mathrm{Ds})$, na profundidade de $\mathbf{0}-\mathbf{0 , 0 5} \mathbf{~ m}$, do Latossolo Vermelho-Amarelo (LVA) e do Cambissolo Háplico (CX), cultivados com cana-de-açúcar ${ }^{(1)}$

\begin{tabular}{|c|c|c|c|c|}
\hline Solo & VTP & Ds & $D_{s} \sigma_{p}$ & $\Delta \mathrm{Ds}$ \\
\hline & $\mathrm{dm}^{3} \mathrm{dm}^{-3}$ & \multicolumn{2}{|c|}{$-\mathrm{kg} \mathrm{dm}^{-3}$} & \\
\hline LVA & $\begin{array}{c}0,48 \mathrm{~B} \\
( \pm 0,01)\end{array}$ & $\begin{array}{c}1,37 \mathrm{~A} \\
( \pm 0,02)\end{array}$ & $\begin{array}{c}1,45 \mathrm{~A} \\
( \pm 0,01)\end{array}$ & $\begin{array}{r}1,07 \mathrm{~A} \\
( \pm 0,01)\end{array}$ \\
\hline $\mathrm{CX}$ & $\begin{array}{c}0,52 \mathrm{~A} \\
( \pm 0,01)\end{array}$ & $\begin{array}{c}1,29 \mathrm{~B} \\
( \pm 0,02)\end{array}$ & $\begin{array}{c}1,39 \mathrm{~B} \\
( \pm 0,02)\end{array}$ & $\begin{array}{r}1,08 \mathrm{~A} \\
( \pm 0,01)\end{array}$ \\
\hline
\end{tabular}

(1) Para cada parâmetro analisado, médias seguidas pelas mesmas letras maiúsculas, nas colunas, não diferem entre si pelo teste de Scott-Knott a $5 \%$ (média de 18 repetições). Números entre parêntese: intervalo de confiança da média. 
Quadro 3. Distribuição temporal dos atributos físicos em decorrência das operações de colheita da cana-deaçúcar, na profundidade de 0-0,05 m, do Latossolo Vermelho-Amarelo (LVA) e do Cambissolo Háplico $(\mathrm{CX})^{(1)}$

\begin{tabular}{|c|c|c|c|c|c|}
\hline \multirow[b]{2}{*}{ Solo } & \multirow[b]{2}{*}{ Antes do tráfego } & \multicolumn{3}{|c|}{ Colheita mecanizada } & \multirow[b]{2}{*}{ Colheita manual ${ }^{(5)}$} \\
\hline & & $11 / 05^{(2)}$ & $03 / 06^{(3)}$ & $08 / 06^{(4)}$ & \\
\hline & \multicolumn{5}{|c|}{ Conteúdo gravimétrico de água (kg kg-1) } \\
\hline LVA & -- & $\begin{array}{c}0,16 \mathrm{~B} \\
\left( \pm 4 \times 10^{-3}\right)\end{array}$ & $\begin{array}{c}0,18 \mathrm{~A} \\
\left( \pm 5 \times 10^{-3}\right)\end{array}$ & $\begin{array}{c}0,11 \mathrm{C} \\
\left( \pm 7 \times 10^{-3}\right)\end{array}$ & $\begin{array}{c}0,19 \mathrm{~A} \\
\left( \pm 4 \times 10^{-3}\right)\end{array}$ \\
\hline \multirow[t]{2}{*}{$\mathrm{CX}$} & -- & $\begin{array}{c}0,22 \mathrm{~B} \\
\left( \pm 6 \times 10^{-3}\right)\end{array}$ & $\begin{array}{c}0,26 \mathrm{~A} \\
\left( \pm 3 \times 10^{-3}\right)\end{array}$ & $\begin{array}{c}0,16 \mathrm{C} \\
\left( \pm 6 \times 10^{-3}\right)\end{array}$ & $\begin{array}{c}0,26 \mathrm{~A} \\
\left( \pm 5 \times 10^{-3}\right)\end{array}$ \\
\hline & \multicolumn{5}{|c|}{ Densidade do solo (Ds) $\left(\mathrm{kg} \mathrm{dm}^{-3}\right)$} \\
\hline LVA & $\begin{array}{c}1,37 \mathrm{D} \\
( \pm 0,02)\end{array}$ & $\begin{array}{c}1,59 \mathrm{~B} \\
( \pm 0,03)\end{array}$ & $\begin{array}{c}1,71 \mathrm{~A} \\
( \pm 0,03)\end{array}$ & $\begin{array}{c}1,43 \mathrm{C} \\
( \pm 0,04)\end{array}$ & $\begin{array}{c}1,32 \mathrm{D} \\
( \pm 0,02)\end{array}$ \\
\hline \multirow[t]{2}{*}{$\mathrm{CX}$} & $\begin{array}{c}1,29 \mathrm{C} \\
( \pm 0,02)\end{array}$ & $\begin{array}{c}1,44 \mathrm{~A} \\
( \pm 0,03)\end{array}$ & $\begin{array}{c}1,47 \mathrm{~A} \\
( \pm 0,01)\end{array}$ & $\begin{array}{c}1,36 \mathrm{~B} \\
( \pm 0,03)\end{array}$ & $\begin{array}{c}1,26 \mathrm{C} \\
( \pm 0,02)\end{array}$ \\
\hline & \multicolumn{5}{|c|}{ Macroporosidade $\left(\mathrm{dm}^{3} \mathrm{dm}^{-3}\right)$} \\
\hline LVA & $\begin{array}{c}0,21 \mathrm{~A} \\
( \pm 0,01)\end{array}$ & $\begin{array}{c}0,10 \mathrm{C} \\
( \pm 0,02)\end{array}$ & $\begin{array}{c}0,05 \mathrm{D} \\
( \pm 0,01)\end{array}$ & $\begin{array}{c}0,16 \mathrm{~B} \\
( \pm 0,02)\end{array}$ & $\begin{array}{c}0,22 \mathrm{~A} \\
( \pm 0,02)\end{array}$ \\
\hline \multirow[t]{2}{*}{$\mathrm{CX}$} & $\begin{array}{c}0,17 \mathrm{~A} \\
( \pm 0,01)\end{array}$ & $\begin{array}{c}0,10 \mathrm{~B} \\
( \pm 0,01)\end{array}$ & $\begin{array}{c}0,07 \mathrm{C} \\
( \pm 0,01)\end{array}$ & $\begin{array}{c}0,14 \mathrm{~B} \\
( \pm 0,02)\end{array}$ & $\begin{array}{c}0,18 \mathrm{~A} \\
( \pm 0,01)\end{array}$ \\
\hline & \multicolumn{5}{|c|}{ Microporosidade $\left(\mathrm{dm}^{3} \mathrm{dm}^{-3}\right)$} \\
\hline LVA & $\begin{array}{c}0,28 \mathrm{~A} \\
( \pm 0,01)\end{array}$ & $\begin{array}{c}0,30 \mathrm{~A} \\
( \pm 0,01)\end{array}$ & $\begin{array}{c}0,30 \mathrm{~A} \\
( \pm 0,01)\end{array}$ & $\begin{array}{c}0,30 \mathrm{~A} \\
( \pm 0,01)\end{array}$ & $\begin{array}{c}0,28 \mathrm{~A} \\
( \pm 0,01)\end{array}$ \\
\hline \multirow[t]{2}{*}{$\mathrm{CX}$} & $\begin{array}{c}0,34 \mathrm{~B} \\
( \pm 0,01)\end{array}$ & $\begin{array}{c}0,35 \mathrm{~B} \\
( \pm 0,01)\end{array}$ & $\begin{array}{c}0,38 \mathrm{~A} \\
( \pm 0,01)\end{array}$ & $\begin{array}{c}0,35 \mathrm{~B} \\
( \pm 0,01)\end{array}$ & $\begin{array}{c}0,33 \mathrm{~B} \\
( \pm 0,01)\end{array}$ \\
\hline & \multicolumn{5}{|c|}{ Diâmetro Médio Geométrico (DMG) (mm) } \\
\hline LVA & $\begin{array}{c}2,35 \mathrm{C} \\
( \pm 0,23)\end{array}$ & $\begin{array}{c}3,73 \mathrm{~A} \\
( \pm 0,59)\end{array}$ & $\begin{array}{c}3,49 \mathrm{~A} \\
( \pm 0,31)\end{array}$ & $\begin{array}{c}2,97 \mathrm{~B} \\
( \pm 0,35)\end{array}$ & $\begin{array}{c}2,32 \mathrm{C} \\
( \pm 0,23)\end{array}$ \\
\hline $\mathrm{CX}$ & $\begin{array}{c}3,95 \mathrm{~A} \\
( \pm 0,11)\end{array}$ & $\begin{array}{c}4,17 \mathrm{~A} \\
( \pm 0,24)\end{array}$ & $\begin{array}{c}4,10 \mathrm{~A} \\
( \pm 0,20)\end{array}$ & $\begin{array}{c}4,26 \mathrm{~A} \\
( \pm 0,05)\end{array}$ & $\begin{array}{c}4,23 \mathrm{~A} \\
( \pm 0,12)\end{array}$ \\
\hline
\end{tabular}

(1) Para cada propriedade analisada, em cada solo, nas diferentes épocas e modalidades de colheita, médias seguidas pelas mesmas letras maiúsculas, nas linhas, não diferem entre si pelo teste de Scott-Knott a 5 \% (média de 18 repetições). (2) Épocas de colheita: novembro de 2005. (3) Março de 2006. (4) Agosto de 2006; Números entre parênteses: intervalo de confiança (IC) da média.

manteve a estrutura inicial. As alterações estruturais dos solos sob tráfego não ocorreram de forma semelhante para todos os atributos, sendo elas o resultado da associação entre as propriedades e as características dos solos em análise (Assis \& Lanças, 2005).

Observa-se (Quadro 3), que a Ds e a macroporosidade do solo se comportaram de maneira inversa, o que se deve à correlação negativa entre esses atributos (Oliveira et al., 2007), sendo as maiores alterações observadas no LVA. Nesse solo, os valores de Ds determinados após a colheita mecanizada da cana-de-açúcar estão relacionados, além da maior suscetibilidade à compactação (Figura 1), com a sua Dsi (Quadro 2).

A colheita mecanizada da cana-de-açúcar aumentou a Ds em qualquer época ou solo (Quadro 3), porém, quando o tráfego ocorreu no mês de agosto de 2006, os aumentos observados neste atributo não são indicativos de compactação adicional. Isso porque os valores de Ds foram inferiores àqueles observados na pressão de preconsolidação (Quadro 2), indicando que a deformação ficou restrita à curva de compressão secundária (Dias Júnior, 1994).
A microporosidade foi alterada apenas no CX e somente quando este foi submetido à colheita mecânica no mês de março de 2006 (Quadro 3). Ressalta-se que, apesar de o aumento na microporosidade, a princípio, ser positivo por resultar em maior retenção de água, ele ocorre à custa da redução de parte dos macroporos (Oliveira et al., 2004). Dessa forma, salienta-se que, em função do relevo em que estão inseridos e devido aos impactos negativos provocados na estrutura do solo, nessas condições de colheita, é esperado um processo erosivo com conseqüências ambientais mais desastrosas no CX, comparado ao LVA.

Os teores de matéria orgânica de cada solo não diferiram pelo teste de Scott-Knott a $5 \%$, independentemente da época ou da modalidade avaliada $\left(15 \pm 0,5\right.$ para o LVA e $27 \pm 0,6 \mathrm{~g} \mathrm{~kg}^{-1}$ para o $\mathrm{CX}$ ), permitindo considerar os efeitos sobre os agregados dos solos como decorrência do tráfego de máquinas durante a colheita mecanizada.

O DMG no LVA aumentou à medida que se aumentou o conteúdo de água no solo durante a colheita mecanizada. Esse fato pode ser explicado pela 
ação da compressão das partículas e pelo aumento do número de contatos entre cada agregado, levando ao seu rompimento e à formação de uma estrutura maciça do solo (Oliveira et al., 2004), sendo este material, portanto, constituído não por agregados, mas por torrões de solo. Nesse caso, o aumento do DMG no LVA indica uma degradação da estrutura. Por outro lado, não houve alteração no DMG do CX, independentemente da época ou da modalidade de colheita, em função do elevado grau de estruturação, tendo reflexos, inclusive, na maior capacidade de suporte de carga (Figura 1).

As alterações estruturais também podem ser verificadas por meio do IHO. As avaliações feitas antes do tráfego, depois da colheita manual e mecanizada, realizada em agosto de 2006 , não provocaram alterações significativas no IHO (Figura 2). Em novembro de 2005 e março de 2006 , ocorreram reduções na amplitude do IHO, o que se deve à degradação estrutural (Figura 2) (Tormena et al., 2007). Com base nesse indicador de qualidade estrutural, observam-se menores reduções no IHO do CX, quando submetido à colheita (Figura $2 b$ ), o que se deve à sua maior resistência à compactação, corroborando os resultados da figura 1 e do quadro 3 .

Torna-se necessária uma análise crítica a respeito do momento em que ocorre alteração nos limites do IHO (Leão et al., 2004). Dessa forma, observa-se que, para o LVA (Figura 2a), a $\theta_{\mathrm{RP}}$ assume o limite inferior do intervalo entre Ds de 1,40 e 1,46 $\mathrm{kg} \mathrm{dm}^{-3}$, valores estes que incluem o intervalo de confiança da $\mathrm{Ds}_{\mathrm{p}}$ $\left(1,45 \pm 0,01 \mathrm{~kg} \mathrm{dm}^{-3}\right.$; Quadro 2). Mesmo com a $\theta_{\mathrm{RP}}$ limitando o crescimento das plantas em conteúdos de água superiores a $\theta_{\mathrm{PMP}}$, as alterações na estrutura, até a Ds de 1,54 kg dm ${ }^{-3}$, não foram suficientes para provocar redução do IHO. O aumento linear na retenção de água na capacidade de campo provoca um deslocamento do IHO, mantendo constante a sua amplitude.

Se, por um lado, a Ds $\sigma_{p}$ do LVA promoveu restrições quanto à resistência à penetração, o valor de Ds de $1,54 \mathrm{~kg} \mathrm{dm}^{-3}$ (Figura 2a) também pode ser considerado um valor de alerta, uma vez que, a partir deste a $\theta_{\mathrm{PA}}$ assume o limite superior do IHO no LVA. As maiores reduções do IHO ocorridas na colheita mecanizada foram observadas em março de 2006, tendo a intersecção dos limites inferiores e superiores do IHO no valor de Ds $1,70 \mathrm{~kg} \mathrm{dm}^{-3}$ (Dsc) (Figura 2a). Observa-se, ainda, que o tráfego de máquinas nessas condições promoveu valores de Ds > Dsc, o que, segundo Tormena et al. (2007), indica severa degradação estrutural nessas condições, restringindo o desenvolvimento dos ciclos posteriores da cana-deaçúcar em qualquer conteúdo de água.

O CX sofreu menor variação total de Ds, quando comparado ao LVA (Figura 2), o que se deve à sua maior resistência à compactação e menor Dsi (Figura 1 e Quadro 2). Até a $\operatorname{Ds}_{\mathrm{p}}(1,39 \pm 0,02$; Quadro 2), o IHO nesse solo assumiu a mesma amplitude da AD, demonstrando a sua adequada qualidade estrutural (Silva et al., 2001) (Figura 2b). Em conteúdos de água no solo superior àqueles encontrados em novembro de 2005, por ocasião do tráfego da colheita, a degradação da estrutura criou limitações ao desenvolvimento da (a)

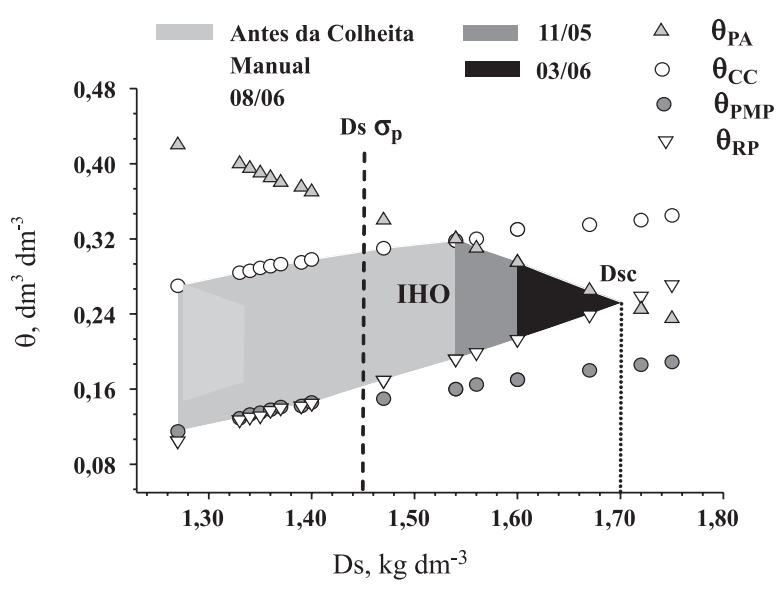

(b)

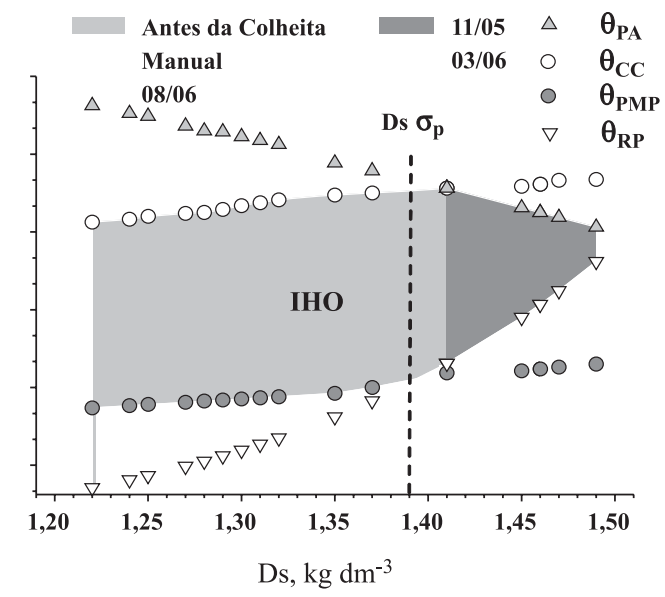

Figura 2. Variação do conteúdo volumétrico de água $\left(\theta, \mathrm{dm}^{3} \mathrm{dm}^{-3}\right)$ com a densidade (Ds), na profundidade de 0-0,05 m, do (a) Latossolo Vermelho-Amarelo (LVA) e (b) Cambissolo Háplico (CX), para os limites críticos de capacidade de campo $\left(\theta_{\mathrm{CC}}\right)$, ponto de murcha permanente $\left(\theta_{\mathrm{PMP}}\right)$, porosidade de aeração $\left(\theta_{\mathrm{PA}}\right)$ e resistência do solo à penetração $\left(\theta_{\mathrm{RP}}\right)$. IHO: intervalo hídrico ótimo; Dsc: densidade crítica ao desenvolvimento das plantas; $D s \sigma_{p}$ : densidade do solo na pressão de preconsolidação. ${ }^{(1)}$ Para épocas ou modalidades de colheita, IHO sob a mesma hachura não diferem entre si, pelo teste de Scott-Knott, a 5 \%. 11/05: novembro de 2005; 3/06: março de 2006; 8/06: agosto de 2006; Manual: colheita manual sem tráfego. 
cultura por oferecer restrições mecânicas ao sistema radicular, mesmo em elevado conteúdo de água no solo, corroborando estudo de Oliveira et al. (2007), além de deficiência na aeração, quando este se encontrar com o conteúdo de água próximo a $\theta_{\text {cc }}$ (Lapen et al., 2004) (Figura 2b). Entretanto, a colheita mecanizada não levou à Dsc, independentemente do conteúdo de água, o que implica menor risco de exposição das culturas, em condições físicas severas, até o desenvolvimento das plantas.

O uso do IHO como indicador de qualidade estrutural do solo para o crescimento das plantas baseia-se no fato de o manejo inadequado do solo alterar vários de seus atributos físicos, que interagem entre si, integrando-os em torno de uma única característica (Silva et al., 2001). Por outro lado, o uso da macroporosidade do solo limitante tem grande aceitação entre os pesquisadores (Centurion et al., 2007; Oliveira et al., 2007). Com base na figura 3, observa-se que a Ds correspondente à macroporosidade de $0,10 \mathrm{dm}^{3} \mathrm{dm}^{-3}\left(1,44\right.$ e $1,59 \mathrm{~kg} \mathrm{dm}^{-3}$, para o CX e LVA, respectivamente) encontras-e dentro da amplitude de IHO ainda considerada positiva (Figura 2), embora, nessas condições, os solos já apresentem restrições quanto à aeração e à resistência à penetração.

A inserção do CX em relevo ondulado, associada à maior suscetibilidade ao encrostamento superficial, em função do maior teor de silte (Brandão et al., 2006) (Quadro 1), faz com que a manutenção da macroporosidade em níveis adequados tenha um papel fundamental no processo de infiltração de água (Sales et al., 1999), pois, além de prejudicar o crescimento e desenvolvimento das plantas, a compactação excessiva potencializará as perdas de solo por erosão (Oliveira et al., 2004). Diante do exposto, sugere-se que a determinação da Ds limitante baseada na

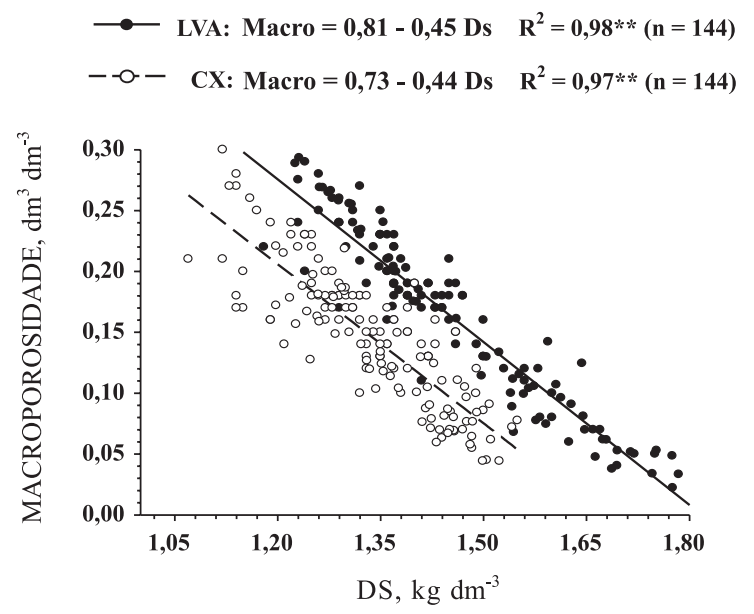

Figura 3. Macroporosidade em função da densidade (Ds), para o Latossolo Vermelho-Amarelo (LVA) e o Cambissolo Háplico (CX), cultivados com cana-de-açúcar. macroporosidade, em contrapartida à Dsc (Tormena et al., 2007), proporciona condições físicas do solo favoráveis à obtenção de produções satisfatórias e garante também a sustentabilidade ambiental.

\section{CONCLUSÕES}

1. O Cambissolo Háplico apresentou maior resistência à compactação que o Latossolo VermelhoAmarelo.

2. A densidade do solo na pressão de preconsolidação foi sensível à ocorrência de alterações estruturais no solo.

3. A macroporosidade do solo limitante apresentou restrições ao crescimento das plantas em níveis de compactação inferiores aos da densidade crítica do intervalo hídrico ótimo.

4. Os valores de densidade do solo correspondente à macroporosidade de $0,10 \mathrm{dm}^{3} \mathrm{dm}^{-3}$ foram $1,44 \mathrm{e}$ $1,59 \mathrm{~kg} \mathrm{dm}^{-3}$ para o Cambissolo Háplico e Latossolo Vermelho-Amarelo, respectivamente.

5. A colheita mecanizada da cana-de-açúcar realizada com conteúdos de água até 0,16 e $0,21 \mathrm{~kg} \mathrm{~kg}^{-1}$, para LVA e CX, respectivamente, não promoveu a compactação excessiva dos solos.

\section{LITERATURA CITADA}

ASSIS, R.L. \& LANÇAS, K.P. Avaliação dos atributos físicos de um Nitossolo Vermelho distroférrico sob sistema plantio direto, preparo convencional e mata nativa. R. Bras. Ci. Solo, 29:515-522, 2005.

BRANDÃO, V.S.; SILVA, D.D.; RUIZ, H.A.; PRUSKI, F.F.; SCHAEFER, C.E.G.R.; MARTINEZ, M.A. \& MENEZES, S.J.M.C. Resistência hidráulica da crosta formada em solos submetidos a chuvas simuladas. R. Bras. Ci. Solo, 30:13-21, 2006.

BUSSCHER, W.J. Adjustment of flat-tipped penetrometer resistance data to common water content. Trans. Am. Soc. Agric. Eng., 33:519-524, 1990.

CAMARGO, O.A. \& ALLEONI, L.R.F. Compactação do solo e o desenvolvimento de plantas. Piracicaba, Escola Superior de Agricultura "Luiz de Queiroz", 1997. 132p.

CENTURION, J.F.; FREDDI, O.S.; ARATANI, R.G.; METZNER, A.F.M.; BEUTLER, A.N. \& ANDRIOLI, I. Influência do cultivo da cana-de-açúcar e da mineralogia da fração argila nas propriedades físicas de Latossolos Vermelhos. R. Bras. Ci. Solo, 31:199-209, 2007.

DIAS JÚNIOR, M.S. Compression of three soils under longterm tillage and wheel traffic. East Lansing, Michigan State University, 1994. 114p. (Tese de Doutorado) 
DIAS JÚNIOR, M.S.; FONSECA, S.; ARAÚJO JÚNIOR, C.F. \& SILVA, A.R. Soil compaction due to forest harvest operations. Pesq. Agropec. Bras., 42:257-264, 2007.

DIAS JÚNIOR, M.S. \& PIERCE, F.J. A simple procedure for estimating preconsolidation pressure from soil compression curves. Soil Technol., 8:139-151, 1995.

EMPRESA BRASILEIRA DE PESQUISA AGROPECUÁRIA . EMBRAPA. Centro Nacional de Pesquisas de Solos. Manual de métodos de análises de solo. 2.ed. Brasília, Produção de Informação, 1997. 212p.

EMPRESA BRASILEIRA DE PESQUISA AGROPECUÁRIA EMBRAPA. Centro Nacional de Pesquisa de Solos. Sistema brasileiro de classificação de solos. 2.ed. Brasília, Produção de Informação, 2006. 306p.

GUEDES, H.M. Efeitos de diferentes sistemas de manejo na distribuição de classes de agregados e nos teores de carbono orgânico em um Latossolo Vermelho-Escuro argiloso na região dos Cerrados. Brasília, Universidade de Brasília, 1997. 153p. (Tese de Mestrado)

KEMPER, W.D. \& ROSENAU, R.C. Aggregate stability and Size distribution. In: KLUTE, A., ed. Methods of soil analysis. Madison, American Society of Agronomy, 1986. Part. 1. p.425-442.

IAIA, A.M.; MAIA, J.C.S. \& KIM, M.E. Uso do penetrômetro eletrônico na avaliação da resistência do solo cultivado com cana-de-açúcar. R. Bras. Eng. Agríc. Amb., 10:523530, 2006 .

KONDO, M.K. \& DIAS JÚNIOR, M.S. Compressibilidade de três Latossolos em função da umidade e uso. R. Bras. Ci. Solo, 23:211-218, 1999.

LAPEN, D.R.; TOPP, G.C.; GREGORICH, E.G. \& CURNOE, W.E. Least limiting water range indicators of soil quality and corn production, Eastern Ontario, Canada. Soil Till. Res., 78:151-170, 2004.

LEÃO, T.P.; SILVA, A.P.; MACEDO, M.C.M.; IMHOFF, S. \& EUCLIDES, V.P.B. Intervalo hídrico ótimo na avaliação de sistemas de pastejo contínuo e rotacionado. R. Bras. Ci. Solo, 28:415-423, 2004.

LEBERT, M. \& HORN, R. A method to predict the mechanical strength of agricultural soils. Soil Till. Res., 19:575-286, 1991.

LEPSCH, I.F., coord. Manual para levantamento utilitário de meio físico e classificação de terras no sistema de capacidade de uso: $4^{\mathrm{a}}$ aproximação. Campinas, Sociedade Brasileira de Ciência do Solo, 1991. 175p.

MELLO, C.R.; OLIVEIRA G.C.; RESCK, D.V.S.; LIMA J.M. \& DIAS JÚNIOR, M.S. Estimativa da capacidade de campo baseada no ponto de inflexão da curva característica. Ci. Agrotec., 26:836-841, 2002.
OLIVEIRA, G.C.; DIAS JÚNIOR, M.S.; RESCK, D.V.S. \& CURI, $\mathrm{N}$. Alterações estruturais e comportamento compressivo de um Latossolo Vermelho distrófico argiloso sob diferentes sistemas de uso e manejo. Pesq. Agropec. Bras., 38:291-299, 2003.

OLIVEIRA, G.C.; DIAS JÚNIOR, M.S.; RESCK, D.V.S. \& CURI, N. Caracterização química e físico-hídrica de um Latossolo Vermelho após vinte anos de manejo e cultivo do solo. R. Bras. Ci. Solo, 28:327-336, 2004.

OLIVEIRA G.C.; SEVERIANO, E.C. \& MELLO, C.R. Dinâmica da resistência à penetração de um Latossolo Vermelho da Microrregião de Goiânia, GO. R. Bras. Eng. Agríc. Amb., 11:265-270, 2007.

RAIJ, B.van. \& QUAGGIO, J.A. Métodos de análise de solo para fins de fertilidade. Campinas, Instituto Agronômico de Campinas, 1983. 16p. (Circular, 63)

RESENDE, M.; CURI, N.; REZENDE, S.B. \& CORRÊA, G.F. Pedologia: Base para distinção de ambientes. 5.ed. Lavras, Universidade Federal de Lavras, 2007. 322p.

SALES, L.E.O.; FERREIRA, M.M.; OLIVEIRA, M.S. \& CURI, N. Estimativa da velocidade de infiltração básica do solo. Pesq. Agropec. Bras., 34:2091-2095, 1999.

SANTOS, R.D.; LEMOS, R.C.; SANTOS, U.G.; KER, J.C. \& ANJOS, L.H.C. Manual de descrição e coleta de solo no campo. 5.ed. Viçosa, MG, Sociedade Brasileira de Ciência do Solo, 2005. 92p.

SILVA, A.P. \& KAY, B.D. Estimating the least limiting water range of soil from properties and management. Soil Sci. Soc. Am. J., 61:977-883, 1997.

SILVA, A.P.; KAY, B.D. \& PERFECT, E. Characterization of the least limiting water range. Soil Sci. Soc. Am. J., 58:1775-1781, 1994.

SILVA, A.P.; TORMENA, C.A. \& IMHOFF, S. Intervalo hídrico ótimo. In: MORAES, M.H.; MÜLLER, M.M.L. \& FOLONI, J.S.S. Qualidade física do solo: Métodos de estudo sistemas de preparo e manejo do solo. Jaboticabal, Funep, 2001. p.1-20.

SNEDECOR, G.W. \& COCHRAN, W.G. Statistical methods. 8.ed. Ames, Iowa State University, 1989. 503p.

SOUZA, Z.M.; PRADO, R.M.; PAIXÃO, A.C.S. \& CESARIN, L.G. Sistemas de colheita e manejo da palhada de canade-açúcar. Pesq. Agropec. Bras., 40:271-278, 2005.

TORMENA, C.A.; ARAÚJO, M.A.; FIDALSKI, J. \& COSTA, J.M. Variação temporal do intervalo hídrico ótimo de um Latossolo Vermelho distroférrico sob sistemas de plantio direto. R. Bras. Ci. Solo, 31:211-219, 2007.

TORMENA, C.A.; SILVA, A.P. \& LIBARDI, P.L. Caracterização do intervalo hídrico ótimo de um Latossolo Roxo sob plantio direto. R. Bras. Ci. Solo, 22:573-581, 1998. 